\title{
Hydrogen-Bond Network Breakage as a First Step to Isopropanol Crystallization
}

\author{
A. Sanz, ${ }^{1}$ M. Jiménez-Ruiz, ${ }^{2}$ A. Nogales, ${ }^{1}$ D. Martín y Marero, ${ }^{2,3}$ and T. A. Ezquerra ${ }^{1}$ \\ ${ }^{1}$ Instituto de Estructura de la Materia, CSIC, Serrano, 121, Madrid 28006, Spain \\ ${ }^{2}$ Institut Laue-Langevin (ILL), BP 156-38042 Grenoble Cedex 9, France \\ ${ }^{3}$ Instituto de Ciencia de Materiales de Aragón, CSIC, Zaragoza, Spain
}

(Received 20 November 2003; published 2 July 2004)

\begin{abstract}
Here we present an experimental study of isopropanol crystallization in real time by means of a novel setup combining simultaneously structural measurements with dynamical techniques. By coupling time resolved neutron diffraction and dielectric spectroscopy experiments we demonstrate that a breakage of the hydrogen-bond network is a precursor step for the crystallization of isopropanol.
\end{abstract}

DOI: 10.1103/PhysRevLett.93.015503

PACS numbers: 61.12.Ex, 61.20.Lc, 77.22.-d

Upon cooling liquids may either crystallize or vitrify. Crystallization is a common phase transition by which molecules self-organize into crystals [1]. Water is one of the most important liquids due to its direct implication in life. Among the peculiarities of liquid water is the presence of a dynamic three-dimensional hydrogen-bond network [2-4]. The physical and chemical properties of hydrogen-bond network forming liquids are controlled by the extension of intermolecular structures resulting from hydrogen bonds $[5,6]$. Related to crystallization, molecular dynamics simulation in water indicated that crystal nucleation occurs after a significant reorganization of the hydrogen-bond network [4]. However, this network modification has not been observed experimentally. This is in part due to the fast freezing kinetics of water [4]. On the contrary, crystallization kinetics of isopropanol, which is also a hydrogen-bond network forming liquid, can be thermally controlled from hours to seconds [7]. The major problem to correlate the evolution of a dynamic magnitude with that of a structural one comes from simultaneity. Crystallization phenomena can be very well studied by diffraction or calorimetry, among others. The dynamics of the hydrogen-bond network can be also well characterized by means of relaxation techniques such as dielectric spectroscopy $[8,9]$. However, in order to find a direct relationship between structure and dynamics, simultaneity may be crucial. Our experiments involve a novel experimental setup which allows one to perform simultaneous measurements of time resolved neutron diffraction (ND) and dielectric spectroscopy (DS). DS measures the complex dielectric permittivity $\varepsilon^{*}=\varepsilon^{\prime}-i \varepsilon^{\prime \prime}$ as a function of frequency, where $\varepsilon^{\prime}$ is the dielectric constant and $\varepsilon^{\prime \prime}$ is the dielectric loss. By measuring as a function of temperature, dielectric spectroscopy becomes a very powerful method to study molecular dynamics in liquids [8-10] above the glass transition temperature $T_{g}$. In this case, molecular motions give rise to a characteristic relaxation process, which can be observed as a maximum [8-10] in $\varepsilon^{\prime \prime}$. To perform simultaneously ND measurements we have designed a special sample holder made from insulating anodized aluminum.
Inside the container the liquid is placed between two standard aluminum pieces acting as electrodes separated by two Teflon ${ }^{\odot}$ spacers and are connected to a Stanford lock-in amplifier SR830 with a dielectric interphase and control unit from Novocontrol. The cell is incorporated into an ILL orange cryostat specially designed to perform neutron scattering. Simultaneous ND and DS experiments were performed in the $\mathrm{D} 1 \mathrm{~B}$ beam line at ILL (Grenoble, France) at a fixed wavelength of $\lambda=0.252 \mathrm{~nm}$. Dehydrated deuterated isopropanol (2propanol d8, 99\% of deuterium ), obtained from SigmaAldrich, was quenched in the glassy state at $75 \mathrm{~K}$ from room temperature. Isothermal crystallization experiments were performed at different temperatures. Crystallization temperatures, $T_{c}$, were reached heating the sample from $75 \mathrm{~K}$ at a heating rate of $0.5 \mathrm{~K} / \mathrm{min}$ up to $T=110 \mathrm{~K}\left(T<T_{g}\right)$ and $2 \mathrm{~K} / \mathrm{min}$ up to $T_{c}$. Figure 1 presents simultaneous ND-DS data taken during the evolution of crystallization at $T_{c}=139 \mathrm{~K}$. The ND intensities are given as a function of the scattering angle $2 \theta$. Every pattern was recorded with an acquisition time of $3 \mathrm{~min}$. The $\varepsilon^{\prime \prime}$ data from DS are presented as a function of frequency $[F=\omega /(2 \pi)$ being $\omega$ the angular frequency]. The initial amorphous state, is characterized by an amorphous halo in the ND diagram and by the primary relaxation process of isopropanol $[8,9]$ in the DS spectrum. As time increases, the onset of crystallization is denoted by the incipient appearance of Bragg peaks in the ND patterns corresponding to the crystalline state of isopropanol [11]. The crystalline amount can be estimated from the ratio between the area below the crystalline peaks to the total scattered area. The primary relaxation (process I) exhibits a decrease of its intensity with crystallization time and a shift towards higher frequencies. The primary relaxation in primary alcohols is attributed to the dynamics of the hydrogen-bond network $[8,9,12]$. This relaxation is accompanied at higher frequencies by a second relaxation (process II) which appears as a high frequency shoulder. The shape analysis of the dielectric curves can be accomplished considering both the primary and the secondary relaxation as being Cole-Davidson processes 


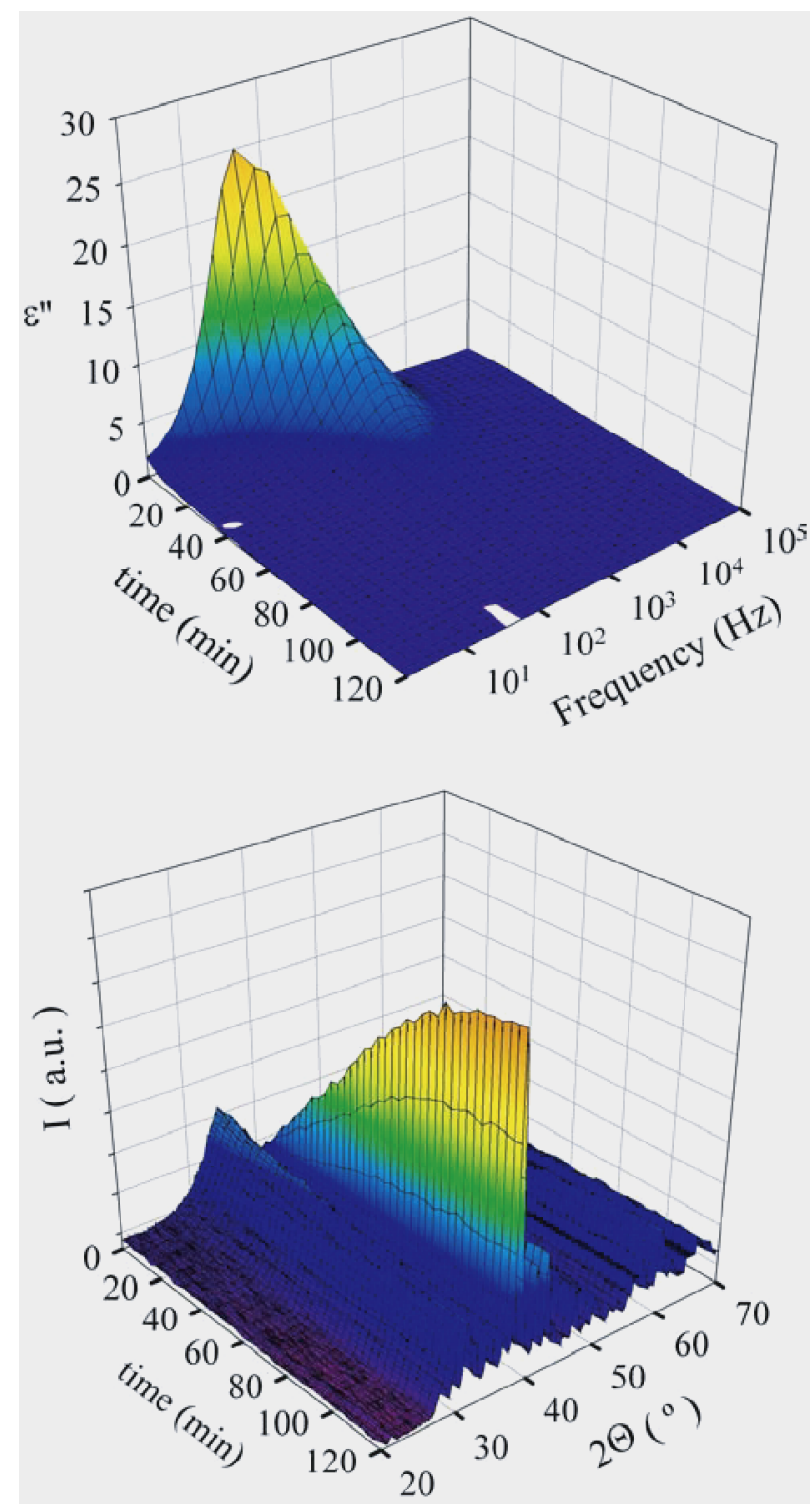

FIG. 1 (color online). Simultaneous neutron diffraction (ND) (bottom) and dielectric loss spectroscopy (DS) (top) experiments as a function of time during an isothermal experiment $(T=139 \mathrm{~K})$. ND patterns for every time are represented as a function of the scattering angle $2 \theta$. DS spectra are given for every time as a function of the frequency.

in accordance with previous reports [8-12]. Accordingly, dielectric loss data can be described as the imaginary part of the dielectric permittivity, $\varepsilon^{\prime \prime}=\operatorname{Im}\left[\varepsilon^{*}\right]=\operatorname{Im}\left[\varepsilon_{\infty}+\right.$ $\left.\sum_{x=\mathrm{III}} \Delta \varepsilon_{x}\left(1+\left(i \omega \tau_{x}\right)\right)^{-c_{x}}\right]$, where $\Delta \varepsilon$ is the relaxation strength which corresponds to the area below the relaxation peak, $\tau_{x}$ is the central relaxation time of the relaxation time distribution function, and $c \leq 1$ is the shape parameter which describes the asymmetric broadening of the relaxation time distribution function [10]. The subscript makes reference either to the primary or to the secondary relaxation. Figure 2 shows as an example two snapshots of the relaxation during the crystallization

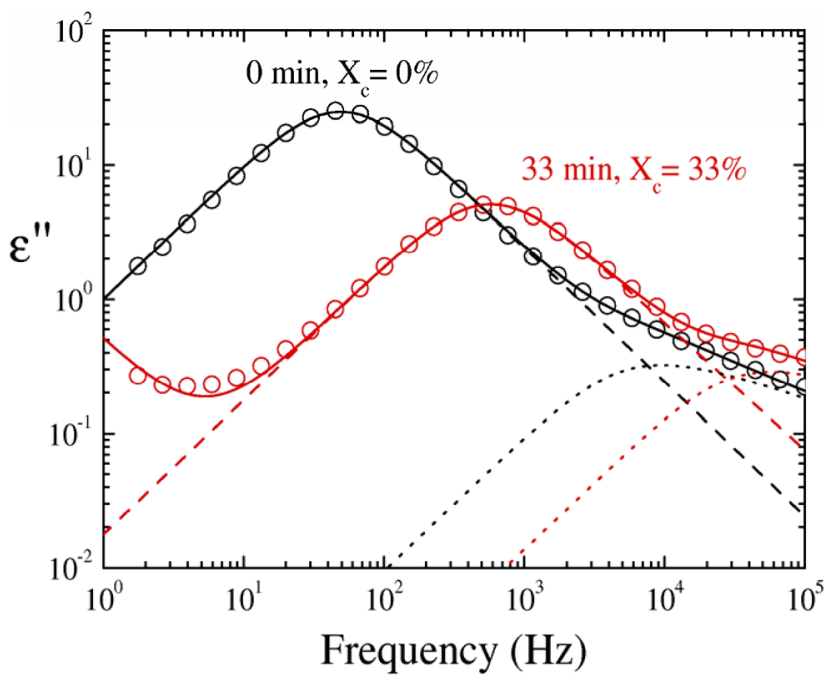

FIG. 2 (color online). Dielectric loss data as a function of frequency at the initial $\left(X_{c}=0 \%\right)$ and at a latter crystallization state $\left(X_{c}=33 \%\right)$ during an isothermal crystallization process at $T=139 \mathrm{~K}$. Dielectric loss data can be described by considering two Cole-Davidson processes: (i) a primary relaxation (I) attributed to the hydrogen-bond network dynamics [8,9], dashed lines, and (ii) a secondary relaxation (II) which is assigned to the $\alpha$ process [8,9], dotted lines.

experiment with the corresponding deconvolution of the different relaxation processes. The initial primary relaxation can be described as being a Debye process $\left(c_{I}=1\right)$ when the sample is less than $20 \%$ crystalline. For higher crystallinity values the shape of the primary relaxation becomes slightly asymmetric $\left(c_{I}=0.7\right)$. As crystallization proceeds an increase in $\varepsilon^{\prime \prime}$ at low frequencies is observed. This effect can be attributed either to the onset of electrical conductivity or to an increment in electrode polarization. In both cases it can be taken into account in the fitting procedure of $\varepsilon^{\prime \prime}$ by considering an additional term $\left(\sigma_{d c} / \varepsilon_{0} \omega\right)^{n}$, where $\sigma_{d c}$ is the electrical conductivity, $\varepsilon_{0}$ is the vacuum permittivity, and the exponent $n$ depends on the conduction mechanism [10]. By this analysis, estimates about the amount of relaxing species involved in every relaxation can be obtained from the area below every relaxation. Figure 3 shows the area $(\Delta \varepsilon)$ below the primary and secondary relaxation as a function of the crystallinity values for two crystallization temperatures. The inset in Fig. 3 shows the dependence of the crystallinity with time during the isothermal crystallization experiments, as obtained from the neutron diffraction patterns acquired in real time. The time scale has been normalized to the characteristic time at which the sample reaches $50 \%$ of crystallinity. From the very early stages of crystallization a dramatic decrease of $\Delta \varepsilon_{\mathrm{I}}$ is observed which can be interpreted as a depletion of the hydrogenbond network since, as mentioned above, $\Delta \varepsilon_{\mathrm{I}}$ is proportional to the amount of species contributing to process I. For example, for $10 \%$ of crystallinity, $\Delta \varepsilon_{\mathrm{I}}$ has decreased 

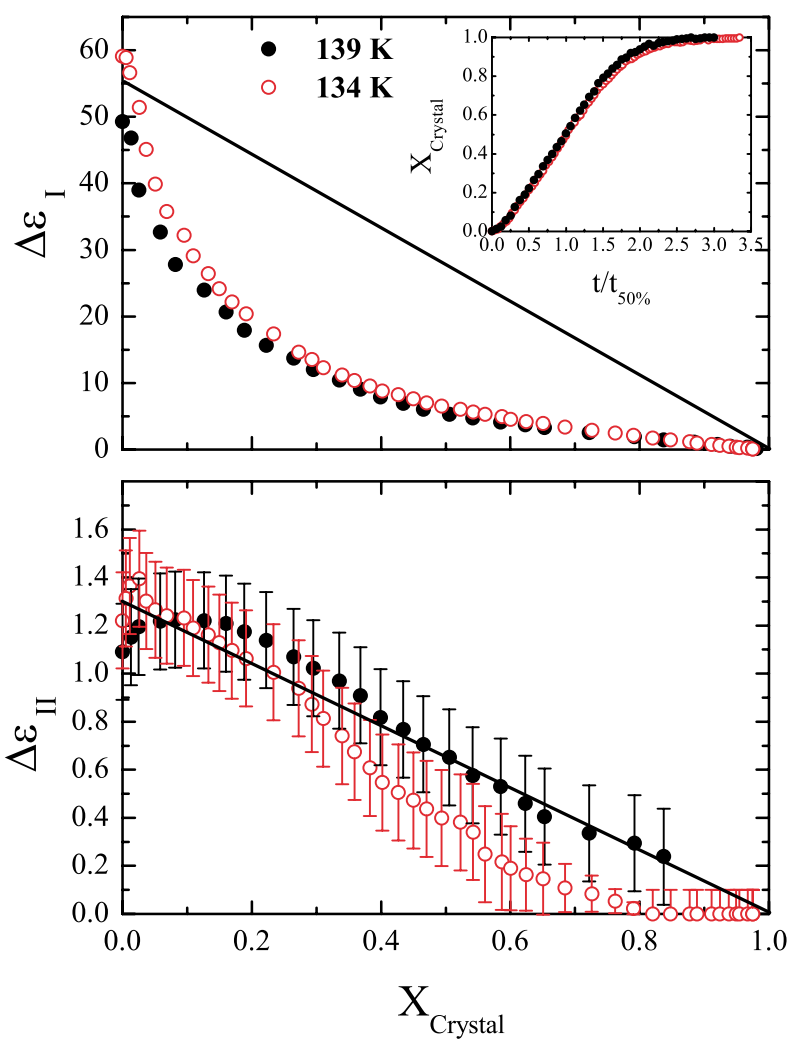

FIG. 3 (color online). Area below every relaxation process for (top) the primary $\left(\Delta \varepsilon_{\mathrm{I}}\right)$ and (bottom) the secondary $\left(\Delta \varepsilon_{\mathrm{II}}\right)$ relaxation processes as a function of crystallinity. Crystallinity values were estimated from the ND patterns as the ratio between the area below the crystalline peaks to the total diffracted intensity for two crystallization temperatures. The straight line correspond to the two-phase model. The inset on the top panel presents the evolution of crystallinty with normalized time for the two studied temperatures.

more than one half of its initial value. Provided that the relaxing species being lost from the network are directly transferred to the crystalline phase in a linear fashion, then a two-phase behavior, denoted in Fig. 3(top) by the continuous line, should be expected. The strong deviation from the two-phase behavior can be interpreted as due to the existence of an intermediate step between the situation in which the molecule is in the network and that in which it occupies a position in the crystal. This stronger than linear reduction of $\Delta \varepsilon_{\mathrm{I}}$ with crystallinity, especially for $X_{c}$ values below 0.1 indicate that the breakage of the hydrogen bond network is a necessary but not sufficient step for nucleation. As suggested by previous simulations [4], in order to form a stable nucleus, appropiate conditions of size and lifetime must be reached by clusters of molecules which, in the present case, have previously left the hydrogen-bond network. Therefore not all the molecules leaving the network, and consequently contributing to the reduction of the primary relaxation, reach simultaneously the conditions for stable nuclei. Once these conditions for long lasting nuclei are met the barriers for subsequent growth are expected to be lower and therefore a weaker reduction of $\Delta \varepsilon_{I}$ with increasing crystallinity is expected as observed in Fig. 3(top) for $X_{c}>0.1$. The dynamics of the hydrogen-bond network can be characterized by $F_{\max }^{\mathrm{I}}$ which represent a mean average frequency of the relaxing species. Figure 4 shows that $F_{\max }^{\mathrm{I}}$ tends to increase in the initial crystallization stages. Both experiments and computer simulations indicate that in hydrogen-bond liquids the primary relaxation time occurs by the wait-and-switch model [12-14]. Here, first a molecule of the network switch its position to reorient itself but it must wait until a favorable condition for reorientation exists in the network. The mean relaxation time, $\tau=1 /\left(2 \pi F_{\max }\right)$ is expected to depend inversely upon the number density of available hydrogen bonding sites [13]. Therefore, in our case, interpreting the decrease of $\Delta \varepsilon_{\mathrm{I}}$ as due to a depletion of the hydrogenbond network is consistent with the parallel increase of the available hydrogen-bond sites as indicated by the increase of $F_{\max }^{\mathrm{I}}$.

Additional information can be added by looking at dependence of $\Delta \varepsilon_{\text {II }}$ with crystallinity [Fig. 3(bottom)]. The secondary process, referred to as $\alpha$ relaxation $[8,9]$,

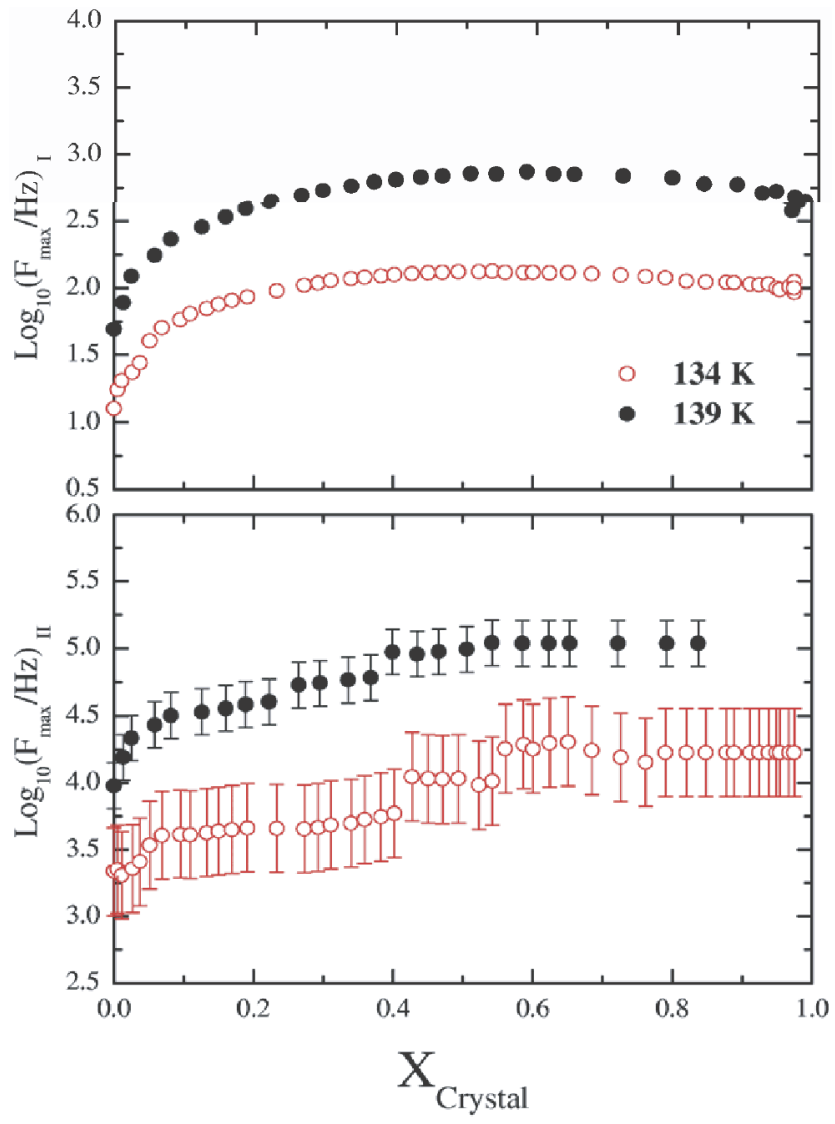

FIG. 4 (color online). Frequency of the dielectric maxima $\left(F_{\max }\right)$ for every relaxation process as a function of crystallinity for two crystallization temperatures. (Top) primary and (bottom) secondary relaxation processes. 
appears as a consequence of the collective motions in the amorphous phase above $T_{g}$. Consequently, $\Delta \varepsilon_{\text {II }}$ is expected to decrease with increasing crystallinity as shown in Fig. 3(bottom). Moreover, in this case the observed decreased is much closer to the two-phase behavior indicating that the relaxing species which are lost in the amorphous phase are almost completely transferred to the crystalline phase. The variation of $F_{\max }^{\mathrm{II}}$ with crystallinity also exhibits an increase (Fig. 4). The dependence of the $\alpha$ relaxation with cross-link point in polymer networks has been investigated in detail $[15,16]$. It was shown that an enhancement of the dynamics occurs when the number of cross-links decreases and consequently the network becomes looser. Because of its inherent dynamic nature, the hydrogen-bond network in primary alcohols is more complex $[17,18]$ than in standard polymer networks. However, the increment of $F_{\max }^{\mathrm{II}}$ with crystallinity can be interpreted consistently as being due to a depletion of relaxing species from the hydrogenbond network. Thus, our experiments indicate that this enhancement of the dynamics may be a requirement previous to the nucleation step.

In conclusion, our crystallization experiments of the hydrogen-bond network forming liquid isopropanol indicate that a breakage of the hydrogen-bond network occurs as a first step previous to the formation of the crystals.

The authors thank the financial support from the MCYT (Grant No. FPA2001-2139), Spain, for generous support of this investigation.

[1] K. F. Kelton, Crystal Nucleation in Liquids and Glasses. Solid State Physics 45 (Academic Press, New York, 1991), pp. 75-177.
[2] I. Ohmine and H. Tanaka, Chem. Rev. 93, 2545 (1993).

[3] H. P. Poole, F. Sciortino, U. Essmann, and H. E. Stanley, Nature (London) 360, 324 (1992).

[4] M. Matsumoto, S. Saito, and I. Ohmine, Nature (London) 416, 409 (2002).

[5] P. Schuster, G. Zundel, and C. Sandorfy, The Hydrogen Bond: Recent Developments in Theory and Experiments (North-Holland, Amsterdam, 1976).

[6] O. Henri-Rousseau and P. Blaise, Adv. Chem. Phys. 103, 1 (1998).

[7] M. Jiménez-Ruiz, T. A. Ezquerra, I. Sics, and M.T. Fernández-Díaz, Appl. Phys. A (Suppl.) 74, S543 (2002).

[8] C. Hansen, F. Stickel, T. Berger, R. Richert, and E.W. Fischer, J. Chem. Phys. 107, 1086 (1997).

[9] A. Kudlik, C. Tschirwitz, S. Benkhof, T. Blochowicz, and E. Rössler, Europhys. Lett. 40, 649 (1997).

[10] F. Kremer and A. Schönhals, Broad Band Dielectric Spectroscopy (Springer, Berlin, 2002).

[11] C. Talón, F. J. Bermejo, C. Cabrillo, G. J. Cuello, J.W. González, J.W. Richardson, Jr., A. Criado, M. A. Ramos, S. Viera, F. L. Cumbrera, and L. M. González, Phys. Rev. Lett. 88, 1155061 (2002).

[12] P. Petong, R. Pottel, and U. Kaatze, J. Phys. Chem. A 103, 6114 (1999).

[13] T. Sato and R. Buchner, J. Chem. Phys. 118, 4606 (2003).

[14] L. Saiz, E. Guàrdia, and J-A. Padró, J. Chem. Phys. 113, 2814 (2000).

[15] C. M. Roland, Macromolecules 27, 4242 (1994).

[16] V. Kramarenko, Yu, T. A. Ezquerra, I. Sics, and F. J. Baltá-Calleja, J. Chem. Phys. 113, 447 (2000).

[17] M. Pagliai, G. Cardini, R. Righini, and V. Schettino, J. Chem. Phys. 119, 6655 (2003).

[18] L. Saiz, E. Guàrdia, and J. A. Padró, J. Chem. Phys. 113, 2814 (2000). 